NMR spectra of Firmianones A, B and C-------The supporting information of "New isoprenylated naphthoquinone dimmer derivatives, firmianones $\mathrm{A}, \mathrm{B}$ and $\mathrm{C}$, from Firmiana plantanifolia (L.f.) Marsili”

Haiyun Bai, Shuo Li, Feng Yin, and Lihong $\mathrm{Hu}^{*}$

We have isolated three novel cytotoxic constituents, named firmianones A(1), B (2) and C (3) from the water fraction of the ethanol extract of the roots of Firmiana plantanifolia (L.f.) Marsili and here are the NMR spectra of them.

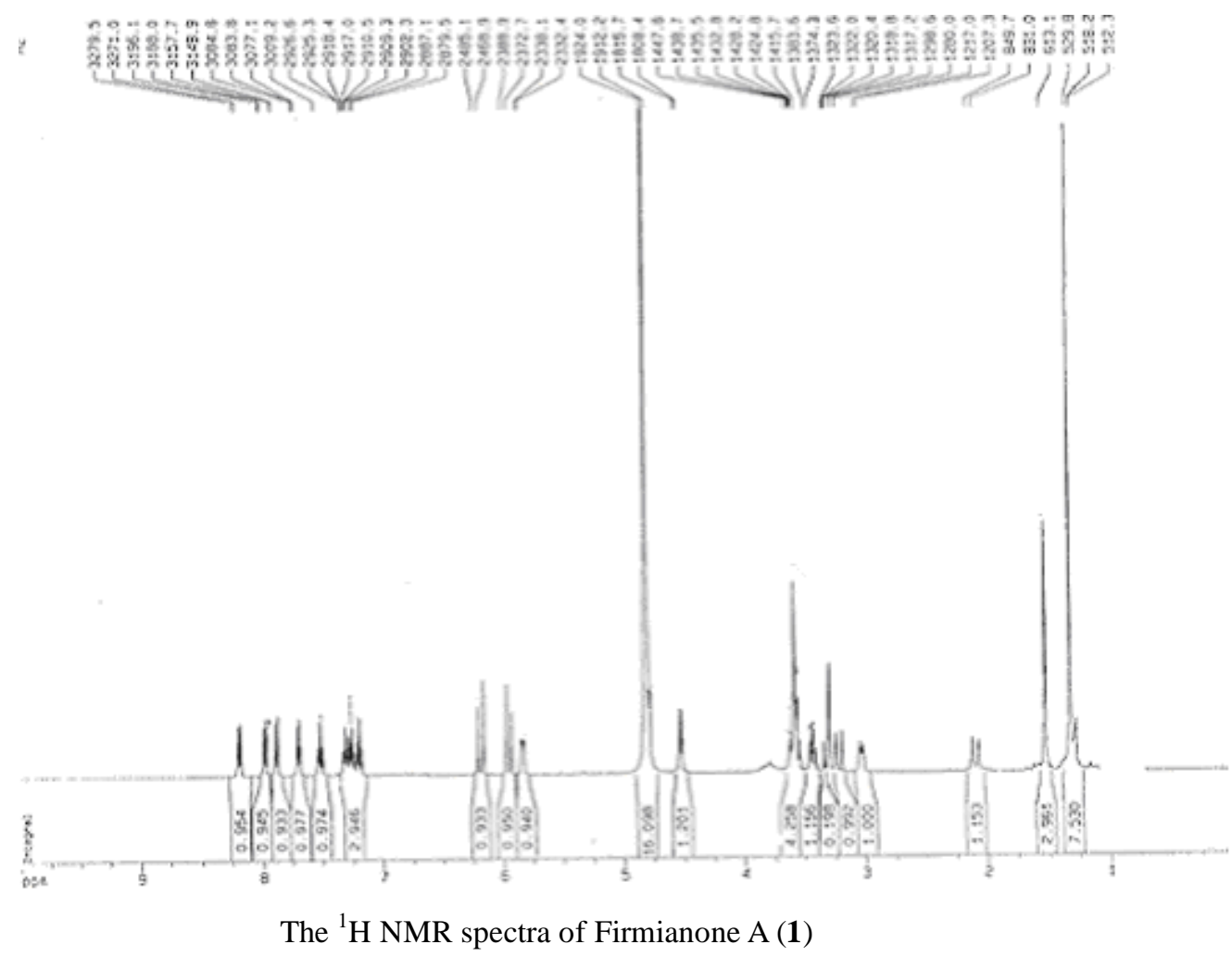




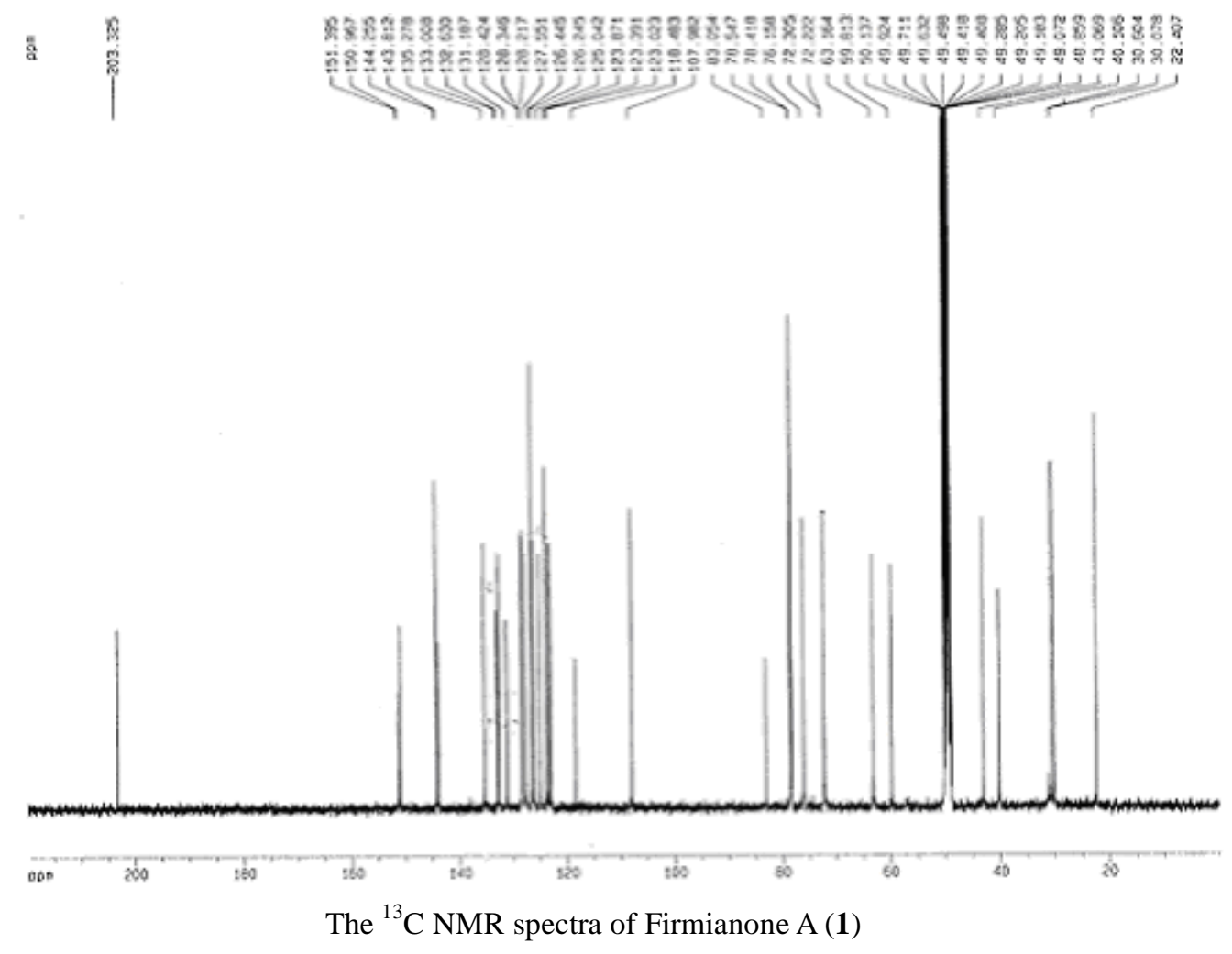




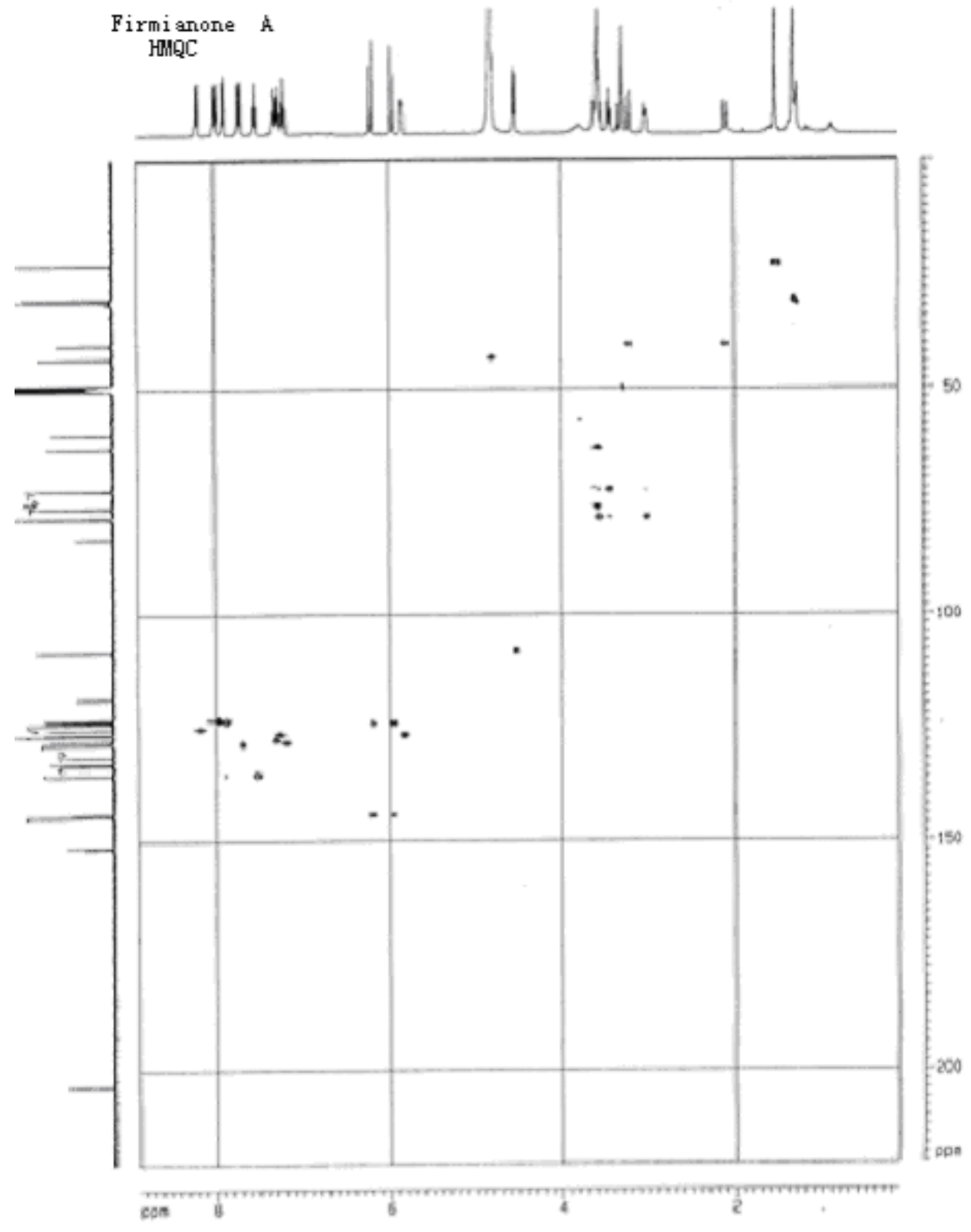

The HMQC spectra of Firmianone A (1) 


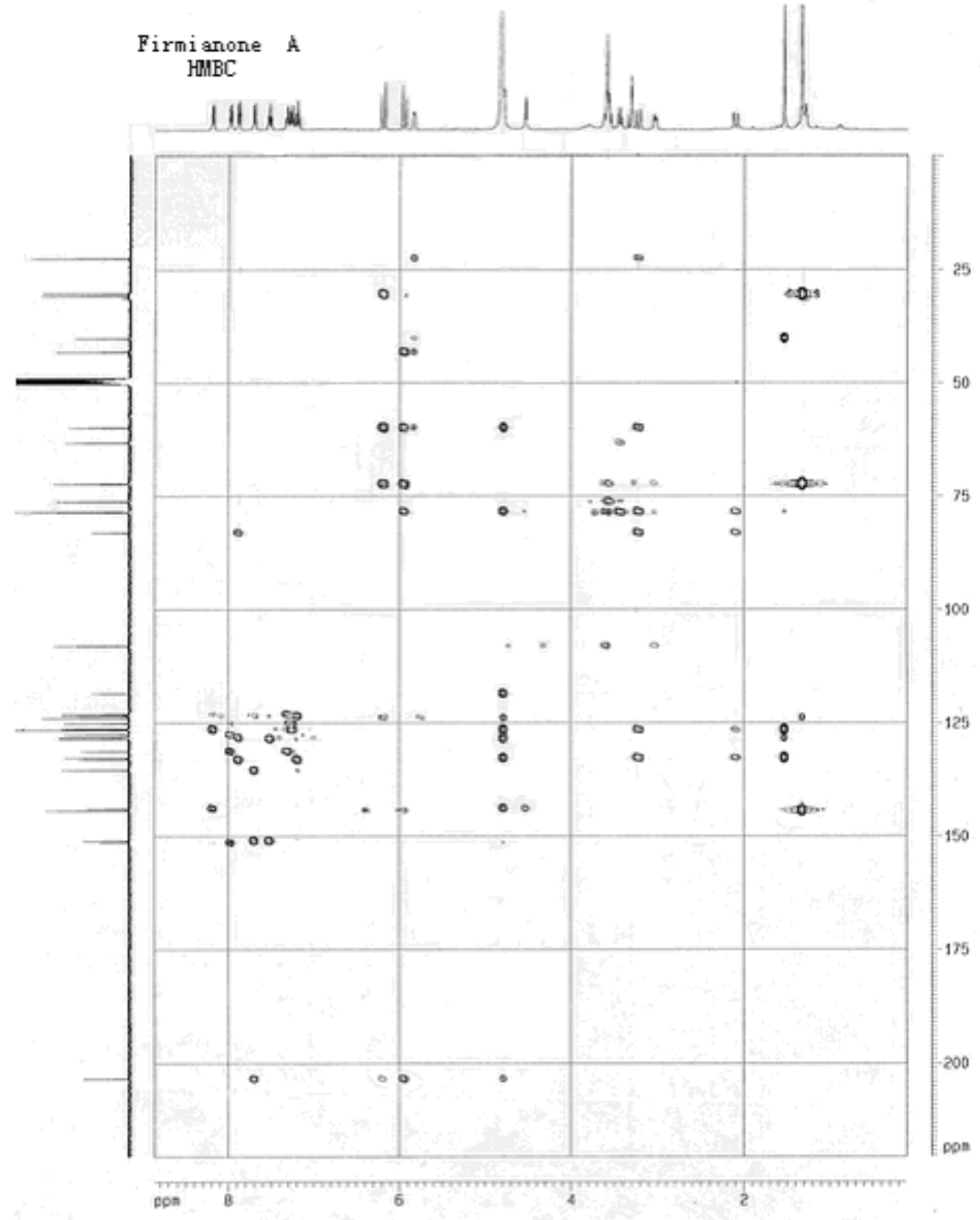

The HMBC spectra of Firmianone A (1) 


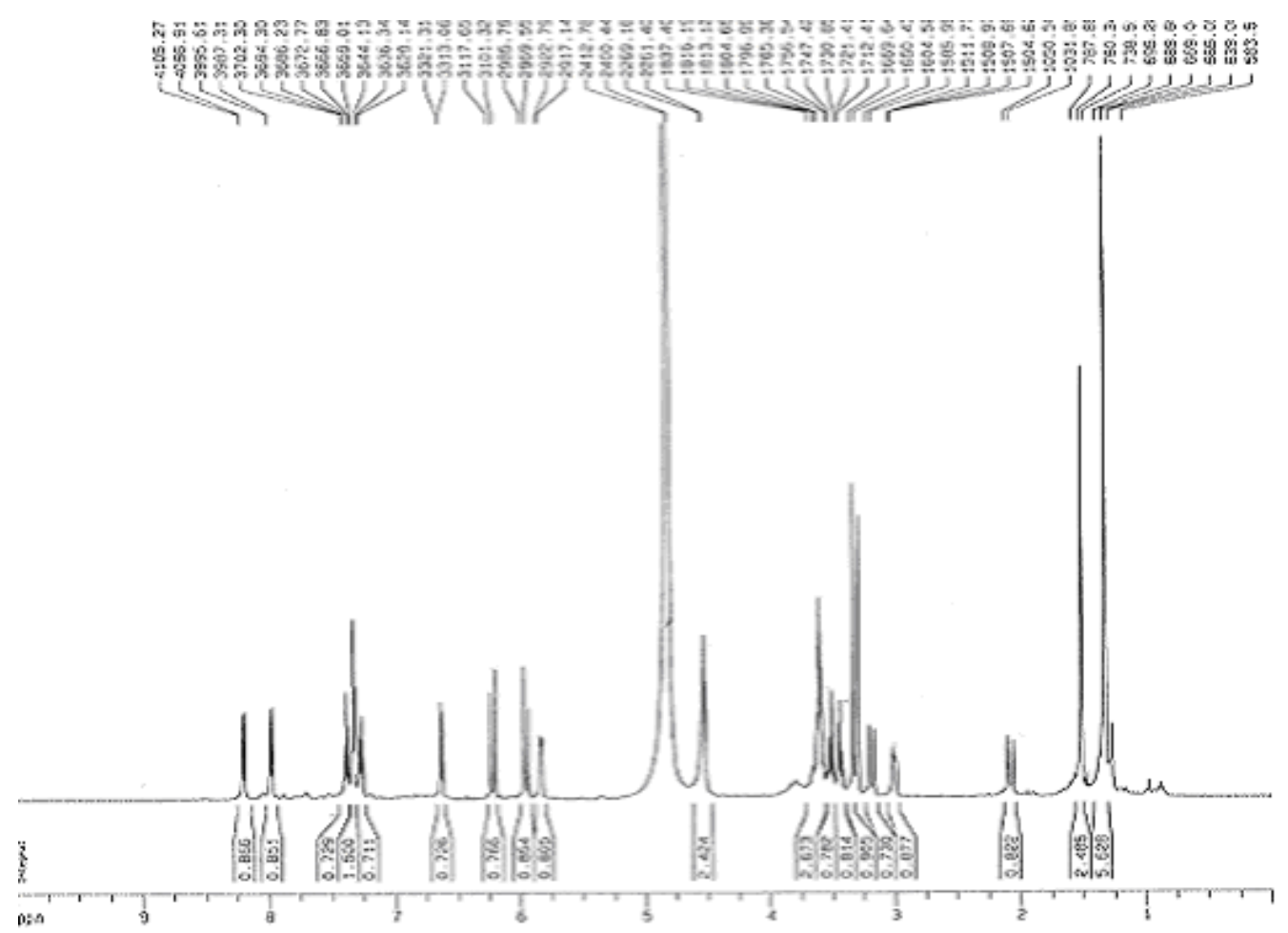

The ${ }^{1} \mathrm{H}$ NMR spectra of Firmianone B (2)

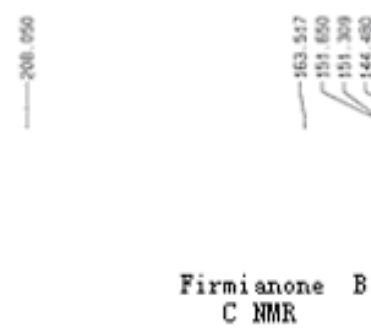

C MMTR

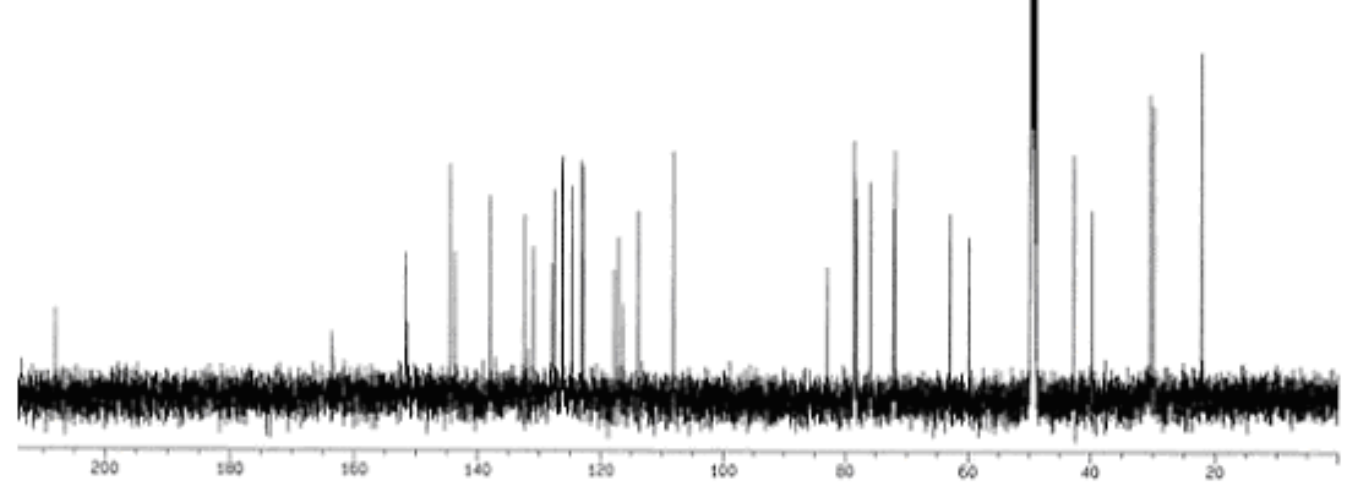

The ${ }^{13}$ C NMR spectra of Firmianone B (2) 


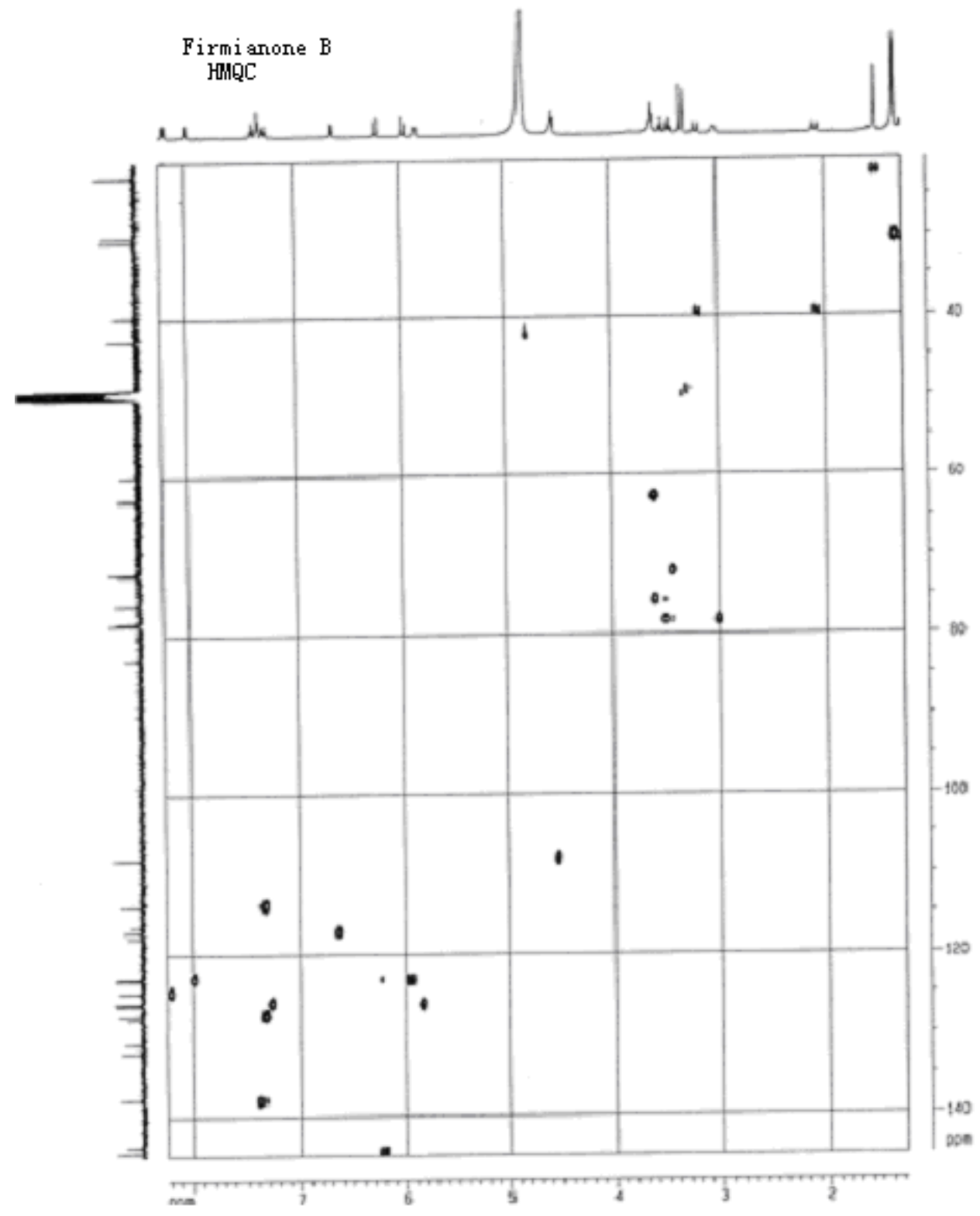

The HMQC spectra of Firmianone B (2) 


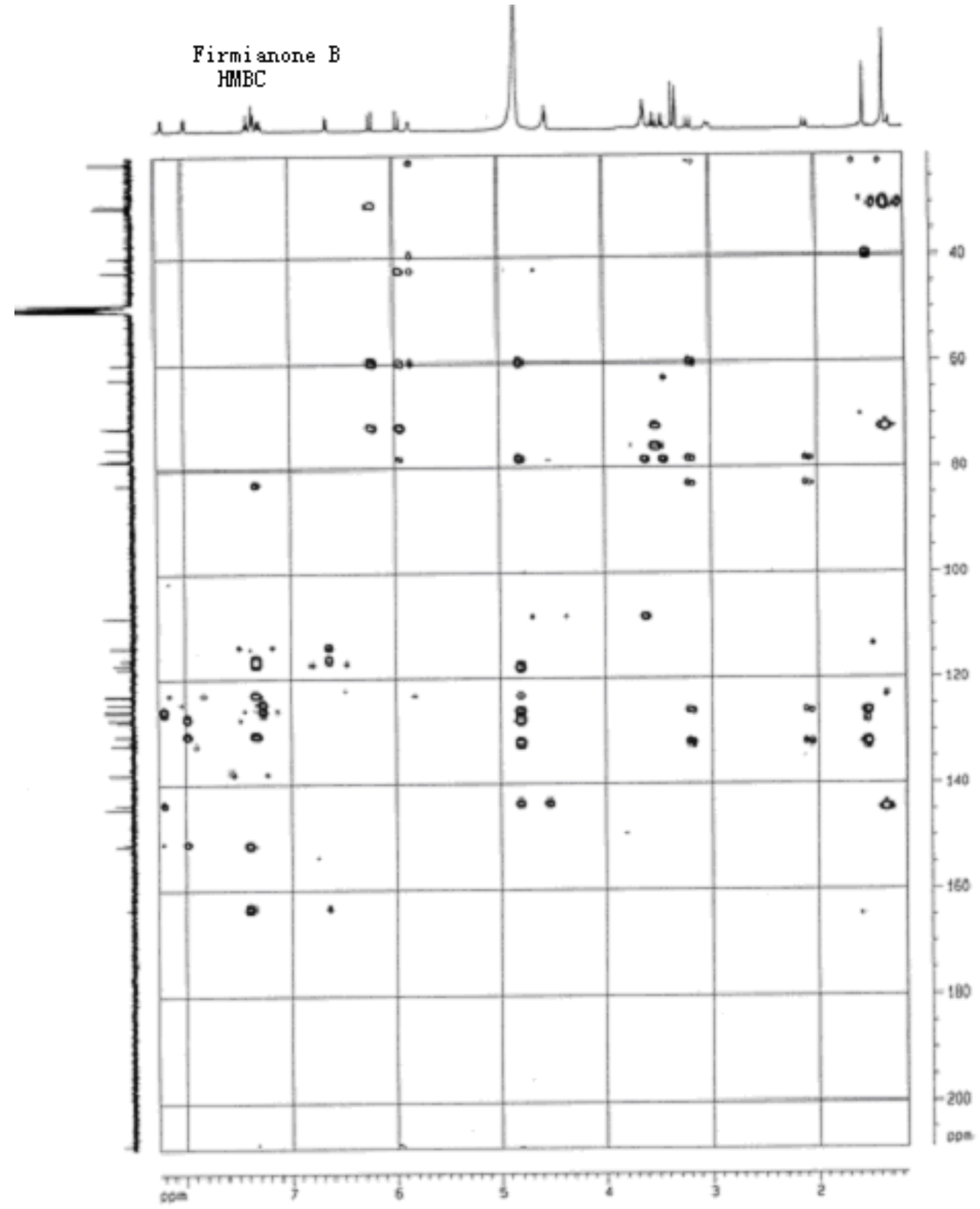

The HMBC spectra of Firmianone B (2) 


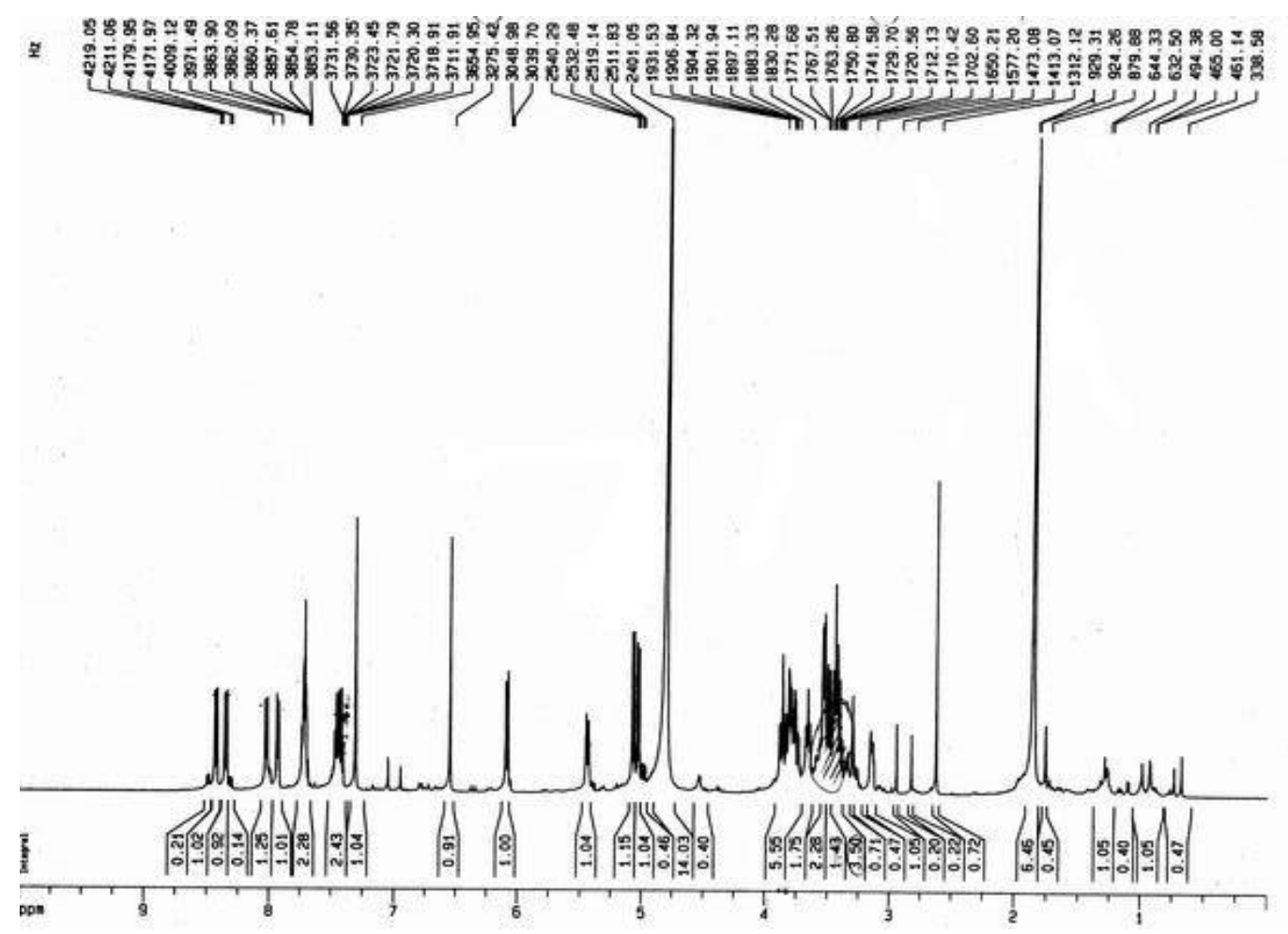

The ${ }^{1} \mathrm{H}$ NMR spectra of Firmianone C (3) 


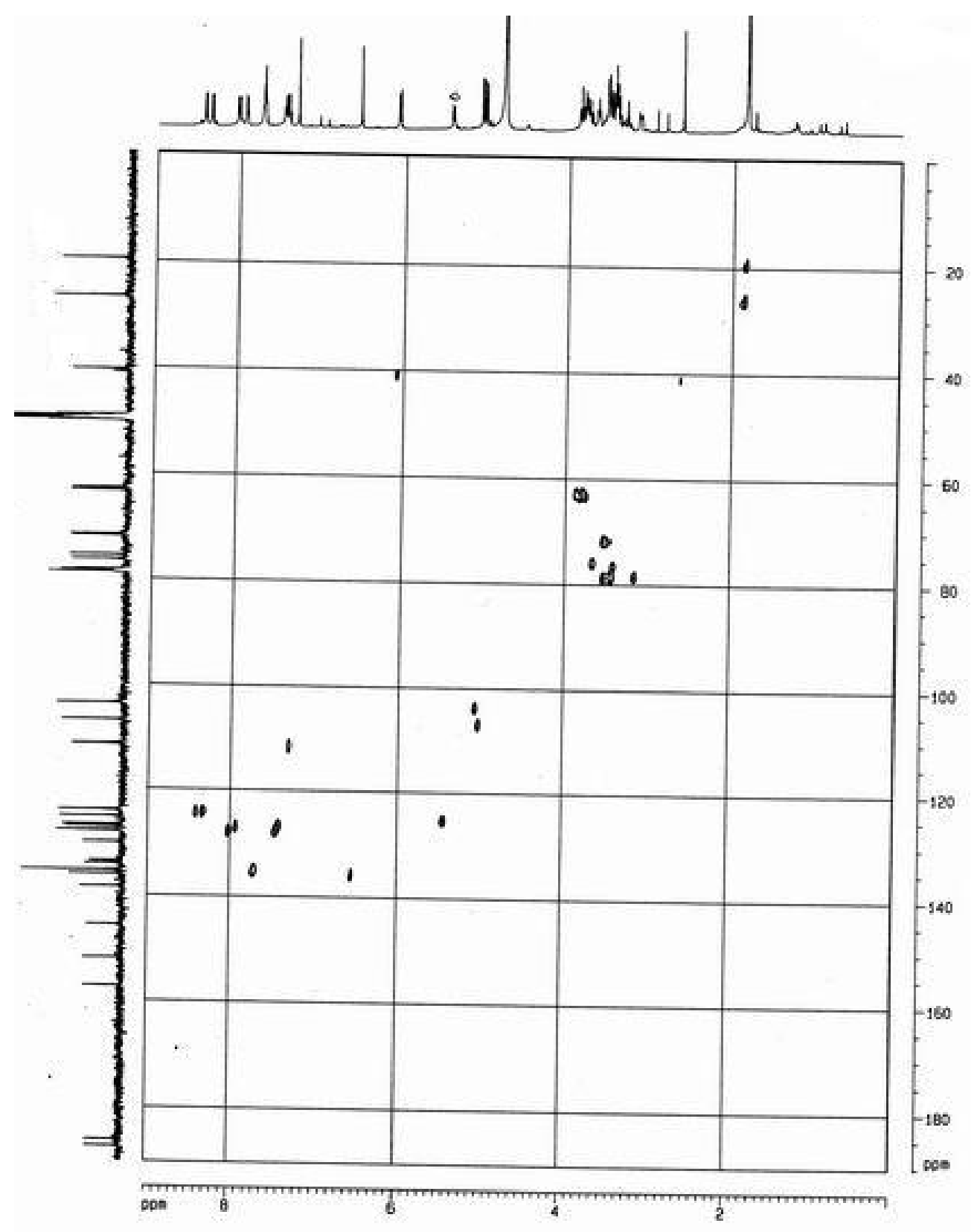

The HMQC spectra of Firmianone C (3) 


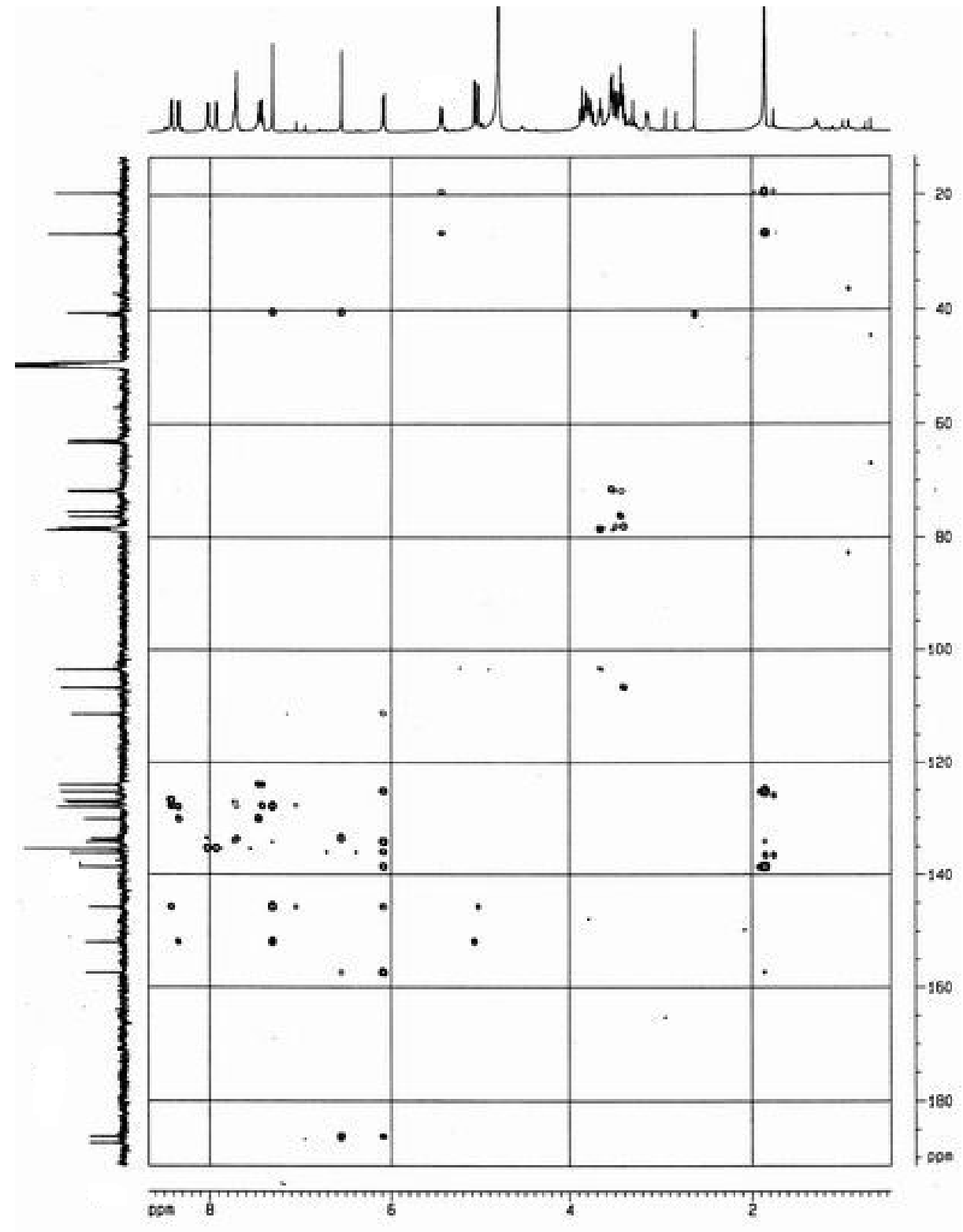

The HMBC spectra of Firmianone C (3) 


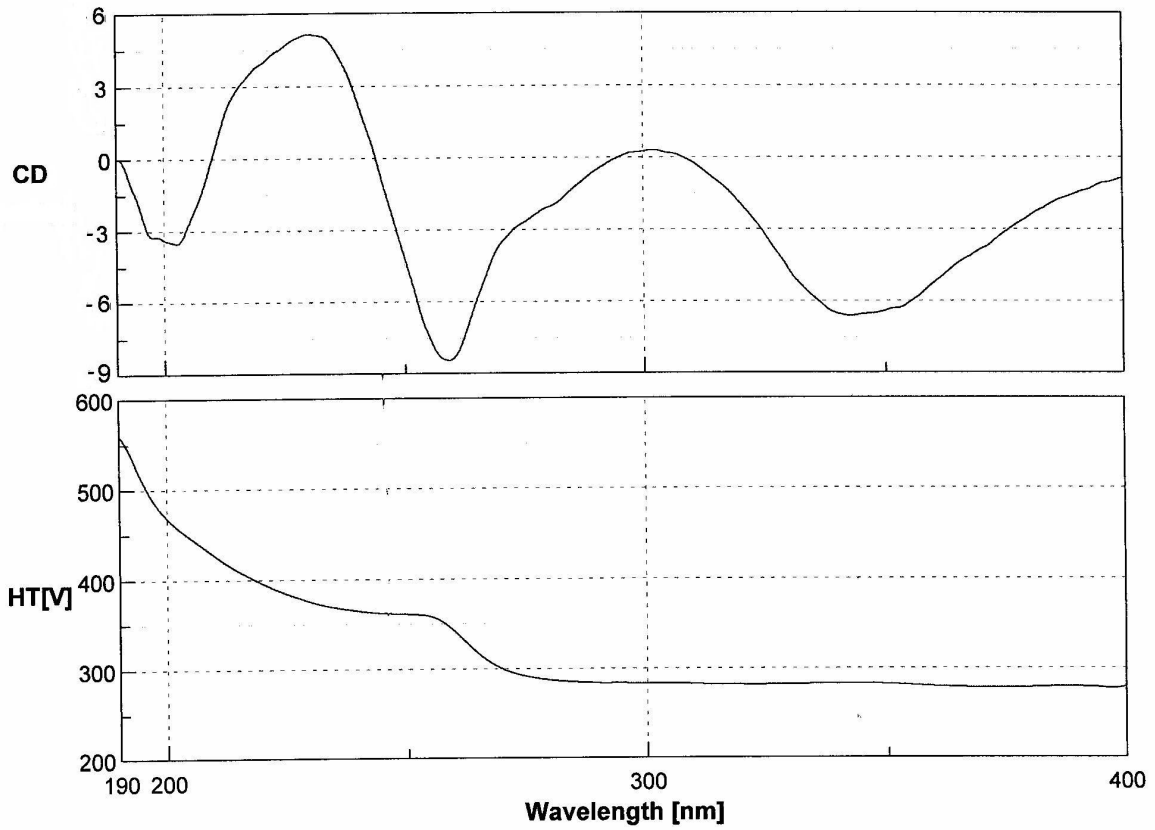

Date

File name

Model

Serial No.

Band width

Response

Sensitivity

Measurement range

Data pitch

Scanning speed

Accumulation

Cell Length

Concentration

Solvent

Temperature

Sample name

Operator

Comment
4/8/04 3:38AM

WT-1 a smooth

J-810

B027760750

$1 \mathrm{~nm}$

$1 \mathrm{sec}$

Standard

$400-190 \mathrm{~nm}$

$0.1 \mathrm{~nm}$

$100 \mathrm{~nm} / \mathrm{min}$.

3

$0.05 \mathrm{~cm}$

$0.5 \mathrm{mg} / \mathrm{L}$

$\mathrm{MeOH}^{\$}$

$20 \mathrm{C}$

WT- 1

Lin

The CD spectra of Firmianone A (1) 

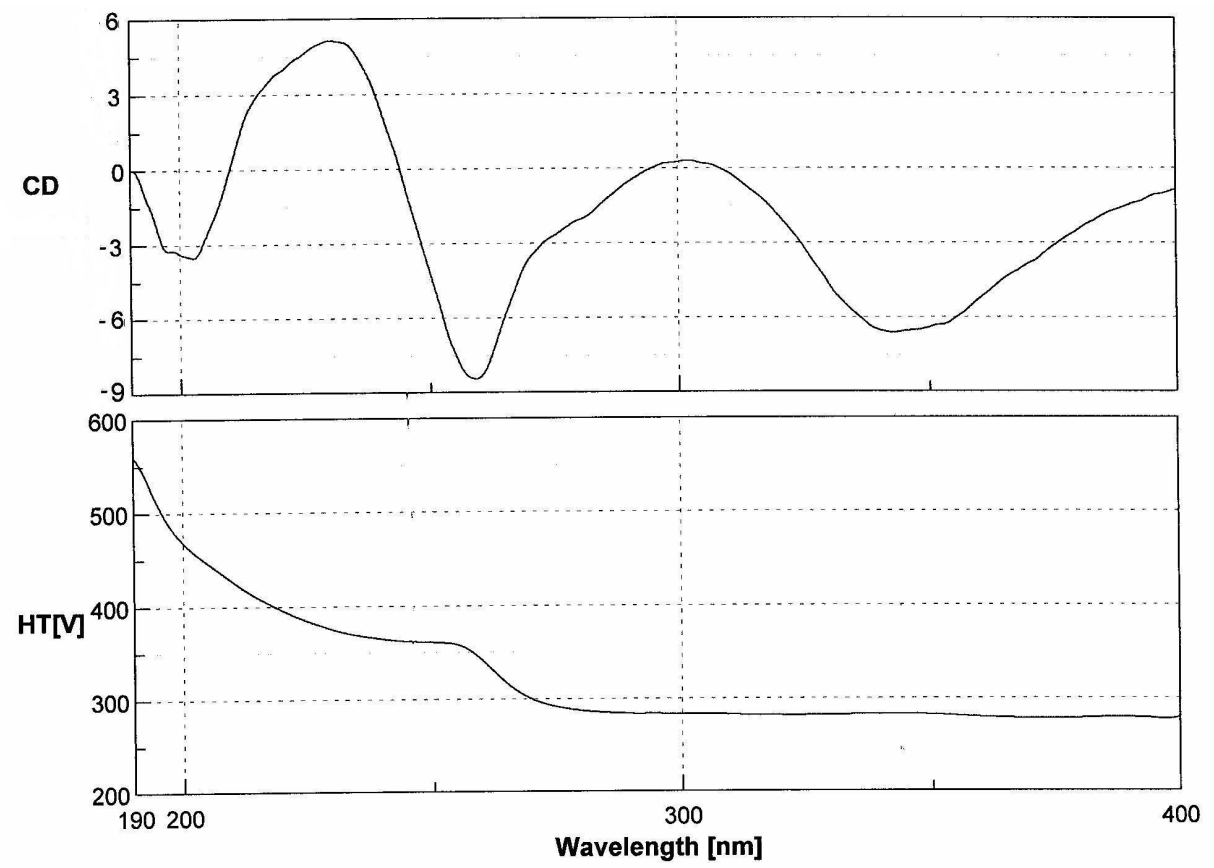

$\begin{array}{ll}\text { Date } & 4 / 8 / 043: 38 \mathrm{AM} \\ \text { File name } & \text { WT-2 a smooth } \\ \text { Model } & \mathrm{J}-810 \\ \text { Serial No. } & \text { B027760750 } \\ \text { Band width } & 1 \mathrm{~nm} \\ \text { Response } & 1 \mathrm{sec} \\ \text { Sensitivity } & \text { Standard } \\ \text { Measurement range } & 400-190 \mathrm{~nm} \\ \text { Data pitch } & 0.1 \mathrm{~nm} \\ \text { Scanning speed } & 100 \mathrm{~nm} / \mathrm{min} \\ \text { Accumulation } & 3 \\ \text { Cell Length } & 0.05 \mathrm{~cm} \\ \text { Concentration } & 0.5 \mathrm{mg} / \mathrm{L} \\ \text { Solvent } & \text { MeOH } \\ \text { Temperature } & 20 \mathrm{C} \\ & \\ \text { Sample name } & \text { WT-2 } \\ \text { Operator } & \text { Lin } \\ \text { Comment } & \end{array}$

The CD spectra of Firmianone B (2) 
Known structures (4-16)

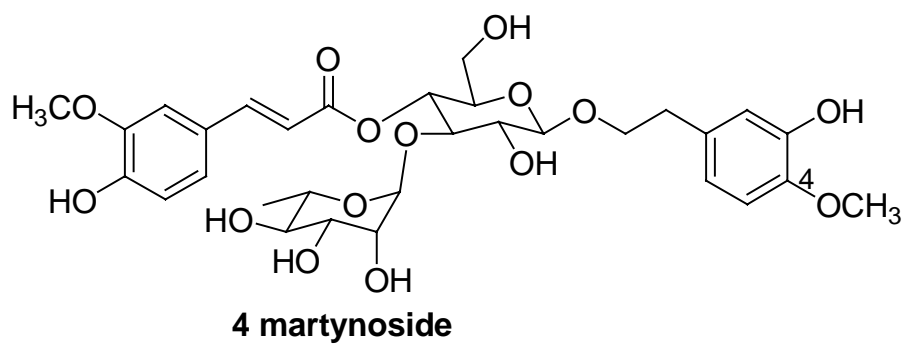<smiles>COc1ccc(/C=C/C(=O)OCCC(O)C(O)C(O)C(O)OC2OC3C(O)C(O)C(O)C(O)C3O2)cc1</smiles>

5 isomartynoside

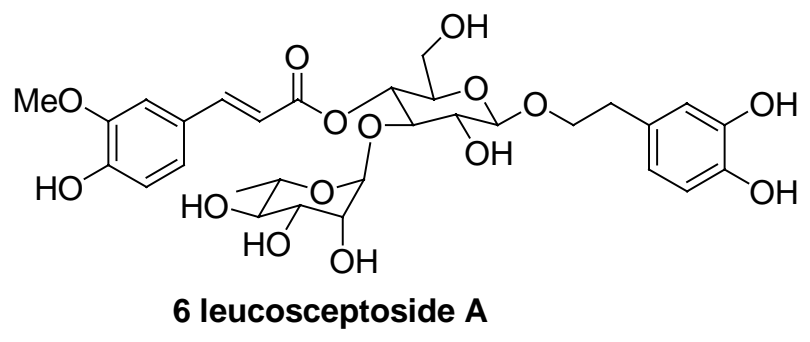

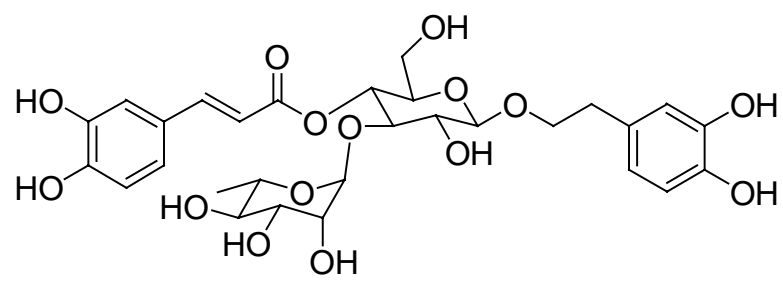

7 acteoside<smiles>O=C(/C=C/c1ccc(O)c(O)c1)OCCC(O)C(O)C(O)OC1OC2(O)OC1C(O)C(O)C2O</smiles>

8 acteoside isomer 


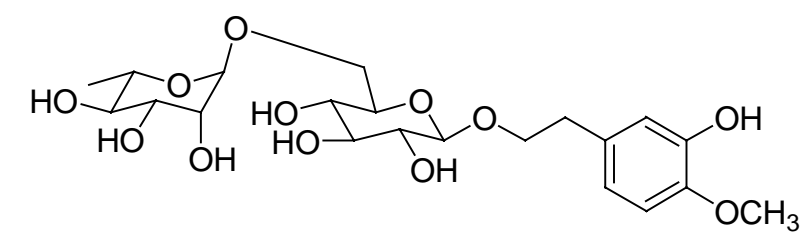

9 forsythoside E<smiles>COc1cc([C@H]2OC[C@@H]3[C@H](c4cc(OC)c(OC)c(OC)c4)OC[C@H]23)cc(OC)c1O</smiles><smiles>CC1(C)CCC2=C(OC1)C(=O)c1ccccc1C2=O</smiles>

11 a-lapachone<smiles>CC1(C)CCC2=C(O1)C(=O)c1c(O)cccc1C2=O</smiles>

12 9-hydroxy-a-lapachone<smiles></smiles>

13 tectol<smiles>COc1ccc([C@@H]2OC[C@H]3[C@H]2CO[C@H]3c2ccc3c(c2)OCO3)cc1O</smiles>

14 paulownin<smiles>c1cc2c(cc1[C@H]1OC[C@H]3[C@H](c4ccc5c(c4)OCO5)OC[C@H]13)OCO2</smiles>

15 sesamin<smiles>COc1cc([C@@H]2OC[C@@H]3[C@H]2CO[C@H]3c2cc(OC)c(OC)c(OC)c2)cc(OC)c1OC</smiles> 


\section{martynoside (4)}

White solid, ${ }^{1} \mathrm{H}$ NMR $\left(300 \mathrm{MHz}, \mathrm{CD}_{3} \mathrm{OD}\right), \delta 7.66(1 \mathrm{H}, \mathrm{d}, J=15.9 \mathrm{~Hz}), 7.20(1 \mathrm{H}, \mathrm{d}$, $J=1.9 \mathrm{~Hz}), 7.08(1 \mathrm{H}, \mathrm{dd}, J=1.9 \mathrm{~Hz} / 8.2 \mathrm{~Hz}), 6.82(1 \mathrm{H}, \mathrm{d}, J=8.2 \mathrm{~Hz}), 6.80(1 \mathrm{H}, \mathrm{d}, J=8.2$ $\mathrm{Hz}), 6.74(1 \mathrm{H}, \mathrm{d}, J=2.0 \mathrm{~Hz}), 6.69(1 \mathrm{H}, \mathrm{dd}, J=2.0 / 8.2 \mathrm{~Hz}), 6.37(1 \mathrm{H}, \mathrm{d}, J=15.9 \mathrm{~Hz}), 5.20$ $(1 \mathrm{H}, \mathrm{d}, J=1.7 \mathrm{~Hz}), 4.92(1 \mathrm{H}, \mathrm{t}, J=9.4 \mathrm{~Hz}), 4.38(1 \mathrm{H}, \mathrm{d}, J=7.9 \mathrm{~Hz}), 3.89(3 \mathrm{H}, \mathrm{s}, \mathrm{OMe})$, $3.82(3 \mathrm{H}, \mathrm{s}, \mathrm{OMe}), 2.83(2 \mathrm{H}, \mathrm{m}), 1.10(3 \mathrm{H}, \mathrm{d}, J=6.2 \mathrm{~Hz}) ;{ }^{13} \mathrm{C} \mathrm{NMR}\left(75 \mathrm{MHz}, \mathrm{CD}_{3} \mathrm{OD}\right)$, $\delta 168.4,150.3,149.4,148.0,147.6,147.3,132.9,127.7,124.5,121.3,117.2,116.7$, $115.3,113.0,112.0,104.2,103.1,81.7,76.2,76.0,73.9,72.4,72.2,71.9,70.7,70.3$, $62.5,56.6,36.6,18.6$

\section{isomartynoside (5)}

White solid, ${ }^{1} \mathrm{H}$ NMR (300 MHz, $\left.\mathrm{CD}_{3} \mathrm{OD}\right), \delta 7.61(1 \mathrm{H}, \mathrm{d}, J=15.9 \mathrm{~Hz}), 7.13(1 \mathrm{H}, \mathrm{d}$, $J=1.9 \mathrm{~Hz}), 7.08(1 \mathrm{H}, \mathrm{dd}, J=1.9 / 8.2 \mathrm{~Hz}), 6.80(1 \mathrm{H}, \mathrm{d}, J=8.2 \mathrm{~Hz}), 6.75(1 \mathrm{H}, \mathrm{d}, J=8.2 \mathrm{~Hz})$, $6.69(1 \mathrm{H}, \mathrm{d}, J=2.0 \mathrm{~Hz}), 6.64(1 \mathrm{H}, \mathrm{dd}, J=2.0 / 8.2 \mathrm{~Hz}), 6.37(1 \mathrm{H}, \mathrm{d}, J=15.9 \mathrm{~Hz}), 5.20(1 \mathrm{H}$, $\mathrm{d}, J=1.0 \mathrm{~Hz}), 4.50(1 \mathrm{H}, \mathrm{m}), 4.38(1 \mathrm{H}, \mathrm{m}), 3.86(3 \mathrm{H}, \mathrm{s}, \mathrm{OMe}), 3.75(3 \mathrm{H}, \mathrm{s}, \mathrm{OMe}), 2.80$ $(2 \mathrm{H}, \mathrm{m}), 1.26(3 \mathrm{H}, \mathrm{d}, J=5.2 \mathrm{~Hz}) ;{ }^{13} \mathrm{C}$ NMR $\left(75 \mathrm{MHz}, \mathrm{CD}_{3} \mathrm{OD}\right), \delta 169.2,150.8,149.6$, 147.6 , 147.1, 147.0, 132.8, 127.7, 124.4, 121.2, 117.2, 116.7, 115.3, 112.8, 112.0, $104.2,103.1,81.6,76.2,75.4,74.2,72.4,72.2,71.9,70.6,70.3,65.0,56.6,36.6,18.1$

\section{leucosceptoside A $(6)$}

White solid, ${ }^{1} \mathrm{H}$ NMR (300 MHz, $\left.\mathrm{CD}_{3} \mathrm{OD}\right), \delta 7.67(1 \mathrm{H}, \mathrm{d}, J=15.9 \mathrm{~Hz}), 7.12(1 \mathrm{H}, \mathrm{d}$, $J=1.9 \mathrm{~Hz}), 7.08(1 \mathrm{H}, \mathrm{dd}, J=1.8 / 8.2 \mathrm{~Hz}), 6.82(1 \mathrm{H}, \mathrm{d}, J=8.2 \mathrm{~Hz}), 6.64-6.75(3 \mathrm{H}, \mathrm{m})$, $6.37(1 \mathrm{H}, \mathrm{d}, J=15.9 \mathrm{~Hz}), 5.23(1 \mathrm{H}, \mathrm{d}, J=1.0 \mathrm{~Hz}), 4.38(1 \mathrm{H}, \mathrm{m}), 3.87(3 \mathrm{H}, \mathrm{s}, \mathrm{OMe}), 2.80$ $(2 \mathrm{H}, \mathrm{m}), 1.16(3 \mathrm{H}, \mathrm{d}, J=6.2 \mathrm{~Hz}) ;{ }^{13} \mathrm{C}$ NMR $\left(75 \mathrm{MHz}, \mathrm{CD}_{3} \mathrm{OD}\right), \delta 168.2,150.8,149.2$, $147.8,146.1,144.0,131.8,127.6,124.2$, 121.2, 117.1, 116.5, 116.3, 115.0, 112.0, $104.2,103.0,81.4,76.0,75.8,74.0,72.4,72.2,72.0,70.6,70.3,62.0,56.6,36.4,18.1$

\section{acteoside (7)}

White solid, ${ }^{1} \mathrm{H}$ NMR (300 MHz, $\left.\mathrm{CD}_{3} \mathrm{OD}\right), \delta 7.57(1 \mathrm{H}, \mathrm{d}, J=15.9 \mathrm{~Hz}), 6.64-7.13(6 \mathrm{H}$, m), $6.37(1 \mathrm{H}, \mathrm{d}, J=15.9 \mathrm{~Hz}), 5.13(1 \mathrm{H}, \mathrm{d}, J=1.0 \mathrm{~Hz}), 4.38(1 \mathrm{H}, J=8.0 \mathrm{~Hz}), 2.80(2 \mathrm{H}$, $J=7.0 \mathrm{~Hz}), 1.16(3 \mathrm{H}, \mathrm{d}, J=6.2 \mathrm{~Hz}) ;{ }^{13} \mathrm{C}$ NMR $\left(75 \mathrm{MHz}, \mathrm{CD}_{3} \mathrm{OD}\right), \delta 168.2,149.3,147.8$, 146.2 , 146.1, 144.0, 131.8, 127.6, 124.2, 121.2, 117.1, 116.4, 116.3, 115.0, 112.0, $104.2,102.9,81.3,75.8,75.4,74.1,72.3,72.2,72.0,70.6,70.3,62.0,36.4,18.1$

\section{acteoside isomer $(8)$}

White solid, ${ }^{1} \mathrm{H}$ NMR $\left(300 \mathrm{MHz}, \mathrm{CD}_{3} \mathrm{OD}\right), \delta 7.54(1 \mathrm{H}, \mathrm{d}, J=15.9 \mathrm{~Hz}), 6.44-7.13(6 \mathrm{H}$, m), $6.27(1 \mathrm{H}, \mathrm{d}, J=15.9 \mathrm{~Hz}), 5.13(1 \mathrm{H}, \mathrm{d}, J=1.0 \mathrm{~Hz}), 4.36(1 \mathrm{H}, J=8.0 \mathrm{~Hz}), 2.77(2 \mathrm{H}$, $J=7.0 \mathrm{~Hz}), 1.26(3 \mathrm{H}, \mathrm{d}, J=6.2 \mathrm{~Hz}) ;{ }^{13} \mathrm{C}$ NMR $\left(75 \mathrm{MHz}, \mathrm{CD}_{3} \mathrm{OD}\right), \delta 168.2,149.3,147.8$, 146.2 , 146.1, 144.0, 131.8, 127.6, 124.2, 121.2, 117.1, 116.4, 116.3, 115.0, 112.0, $104.2,102.9,84.3,75.5,75.2,74.1,72.3,72.2,72.0,70.4,70.2,65.0,36.4,18.1$

\section{forsythoside $\mathbf{E}$ (9)}

White solid, ${ }^{1} \mathrm{H}$ NMR (300 MHz, $\left.\mathrm{CD}_{3} \mathrm{OD}\right), \delta 6.44-6.93(3 \mathrm{H}, \mathrm{m}), 4.73(1 \mathrm{H}, \mathrm{d}, J=1.2$ $\mathrm{Hz}), 4.26(1 \mathrm{H}, \mathrm{d}, J=8.0 \mathrm{~Hz}), 2.77(2 \mathrm{H}, J=7.0 \mathrm{~Hz}), 1.26(3 \mathrm{H}, \mathrm{d}, J=6.0 \mathrm{~Hz}) ;{ }^{13} \mathrm{C} \mathrm{NMR}$ (75 MHz, $\left.\mathrm{CD}_{3} \mathrm{OD}\right), \delta 146.3,145.0,130.8,120.4$, 117.6, 116.4, 104.2, 102.1, 78.3, 76.5, $74.8,73.7,72.5,72.0,71.6,71.2,70.4,62.0,36.4,18.1$

\section{(+)-syringaresinol- $\boldsymbol{O}$ - $\beta$-D-glucopyranoside (10)}

White power, ${ }^{1} \mathrm{H}$ NMR $\left(300 \mathrm{MHz}, \mathrm{CD}_{3} \mathrm{OD}\right), \delta 6.65(2 \mathrm{H}, \mathrm{s}), 6.60(2 \mathrm{H}, \mathrm{s}), 3.70(6 \mathrm{H}, \mathrm{s}$, $4 \times \mathrm{OMe}), 3.64-4.26(4 \mathrm{H}, \mathrm{m}) ;{ }^{13} \mathrm{C} \mathrm{NMR}\left(75 \mathrm{MHz}, \mathrm{CD}_{3} \mathrm{OD}\right), \delta 152.6,148.0,137.2$, 
135.2, 134.1, 131.4, 104.4, 104.0, 102.9, 85.3, 85.1, 77.3, 76.5, 74.2, 71.2, 70.1, 62.0, 53.6

\section{$\alpha$-lapachone (11)}

Yellow power, ${ }^{1} \mathrm{H}$ NMR $\left(300 \mathrm{MHz}, \mathrm{CDCl}_{3}\right), \delta 8.58(1 \mathrm{H}, \mathrm{d}, J=7.2 \mathrm{~Hz}), \delta 8.20(1 \mathrm{H}, \mathrm{d}$, $J=7.2 \mathrm{~Hz}), 7.60(2 \mathrm{H}, \mathrm{t}, J=7.2 \mathrm{~Hz}), 2.10(2 \mathrm{H}, \mathrm{m}), 1.64(2 \mathrm{H}, \mathrm{m}), 1.30(6 \mathrm{H}, \mathrm{s})$

\section{9-hydroxy- $\alpha$-lapachone (12)}

Yellow power, ${ }^{1} \mathrm{H}$ NMR $\left(300 \mathrm{MHz}, \mathrm{CDCl}_{3}\right), \delta 8.58(1 \mathrm{H}, \mathrm{d}, J=7.2 \mathrm{~Hz}), \delta 8.20(1 \mathrm{H}, \mathrm{d}$, $J=7.2 \mathrm{~Hz}), 7.60(2 \mathrm{H}, \mathrm{t}, J=7.2 \mathrm{~Hz}), 2.10(2 \mathrm{H}, \mathrm{m}), 1.64(2 \mathrm{H}, \mathrm{m}), 1.30(6 \mathrm{H}, \mathrm{s})$

\section{Tectol (13)}

Yellow power, ${ }^{1} \mathrm{H}$ NMR $\left(300 \mathrm{MHz}, \mathrm{CDCl}_{3}\right), \delta 8.22(2 \mathrm{H}, \mathrm{m}), 8.17(2 \mathrm{H}, \mathrm{m}), 7.52(2 \mathrm{H}$, m), $7.49(2 \mathrm{H}, \mathrm{m}), 5.91(2 \mathrm{H}, \mathrm{d}, J=9.9 \mathrm{~Hz}), 5.61(2 \mathrm{H}, \mathrm{d}, J=9.9 \mathrm{~Hz}), 1.52(6 \mathrm{H}, s, 2 \times$ $\mathrm{OMe}), 1.47(6 \mathrm{H}, s, 2 \times \mathrm{OMe}) ;{ }^{13} \mathrm{C} \mathrm{NMR}\left(75 \mathrm{MHz}, \mathrm{CDCl}_{3}\right), \delta$ 145.6, 142.5, 131.1, $126.9,1236.7,126.3,126.2,123.6,122.4,121.7,76.4$

\section{Paulownin (14)}

White power, ${ }^{1} \mathrm{H}$ NMR $\left(300 \mathrm{MHz}, \mathrm{CDCl}_{3}\right), \delta 6.94(1 \mathrm{H}, \mathrm{m}), 6.09(2 \mathrm{H}, \mathrm{m}), 5.86(1 \mathrm{H}$, $\mathrm{dd}, J=6.0 / 9.0 \mathrm{~Hz}), 5.39(1 \mathrm{H}, \mathrm{dd}, J=6.0 / 9.0 \mathrm{~Hz}), 5.23(1 \mathrm{H}, s), 5.15(1 \mathrm{H}, \mathrm{d}, J=4.8 \mathrm{~Hz})$, $4.06(4 \mathrm{H}, s), 3.07-3.20(6 \mathrm{H}, m) ;{ }^{13} \mathrm{C}$ NMR $\left(75 \mathrm{MHz}, \mathrm{CDCl}_{3}\right), \delta 148.2,147.3,134.8$, $129.4,120.1,119.8,108.6,108.2,107.5,106.9,101.2,101.1,91.7,87.5,85.9,74.9$, $71.6,60.6$

\section{Sesamin (15)}

White power, ${ }^{1} \mathrm{H}$ NMR (300 MHz, $\left.\mathrm{CDCl}_{3}\right), \delta 6.94(1 \mathrm{H}, \mathrm{m}), 6.95(2 \mathrm{H}, \mathrm{m}), 6.16(2 \mathrm{H}$, $\mathrm{dd}, J=4.0 / 9.0 \mathrm{~Hz}), 5.79(1 \mathrm{H}, \mathrm{dd}, J=7.2 / 9.0 \mathrm{~Hz}), 5.29(1 \mathrm{H}, \mathrm{d}, J=4.5 \mathrm{~Hz}), 4.06(4 \mathrm{H}, s)$, 3.10-3.30 (6H, $m) ;{ }^{13} \mathrm{C}$ NMR (75 MHz, $\left.\mathrm{CDCl}_{3}\right), \delta 148.5,147.1$ 134.6, 129.3, 120.1, $119.8,108.6,108.2,107.5,106.9,101.2,101.1,87.5,85.9,74.9,71.6,63.5,60.6$

\section{Yangambin (16)}

White power, ${ }^{1} \mathrm{H}$ NMR $\left(300 \mathrm{MHz}, \mathrm{CDCl}_{3}\right), \delta 6.58(4 \mathrm{H}, \mathrm{s}), 4.75(2 \mathrm{H}, \mathrm{d}, J=5.4 \mathrm{~Hz})$, 4.22-4.40 (2H, m), $3.86(12 \mathrm{H}, \mathrm{s}), 3.83(6 \mathrm{H}, \mathrm{s}), 3.82-3.97(2 \mathrm{H}, \mathrm{m}) ;{ }^{13} \mathrm{C}$ NMR $(75 \mathrm{MHz}$, $\left.\mathrm{CDCl}_{3}\right), \delta 154.0,137.6,136.7,103.0,85.9,72.0,60.0,56.1,54.3$ 